\title{
SwissDRG zwingt Spitäler zur Produktivitätssteigerung
}

\section{Thomas Kaufmann ${ }^{a}$, Guido Schüpfer ${ }^{b}$}

a Dr. med., Gesamtleiter DRG \& Qualitäts- und Riskmanagement, Luzerner Kantonsspital (LUKS);

b PD Dr. med., Leiter Stab Medizin \& Co-Chefarzt Anästhesie, Luzerner Kantonsspital (LUKS)

Jede neue DRG-Version führt zu teils dramatischer Neubewertung der Fallpauschalen. Bisher kaum beachtet wurde, dass nicht nur durch die normierte Abgeltung einzelner DRGs, sondern auch durch die im System definierte Normliegedauer ein deutlicher Produktivitätsdruck erreicht wird. Durch diese Anpassung sehen sich viele Kliniken mit empfindlichen Ertragseinbussen konfrontiert.

Seit dem 1. Januar 2012 werden in der Schweiz stationäre Spitalleistungen durch Fallpauschalpreise nach SwissDRG vergütet. Die gut 10000 Diagnosen und 20000 Therapien werden einer von etwas über 1000 DRG-Gruppen zugeteilt. Jede DRG erhält ein Kostengewicht, welches multipliziert mit der für jedes Spital individuellen Baserate (Basispreis) den Preis der Leistung ergibt. Das durchschnittliche Fallgewicht aller Behandlungen in der Schweiz sollte ein Kostengewicht von 1,0 erhalten. Das ist nicht zu erreichen, weil weniger als 20\% der Fälle für die Berechnungen beigezogen werden. Zudem werden nicht plausible Fälle gelöscht, um Ausreisser bezüglich Kosten zu eliminieren. Dies führt zur Senkung des Mittelwertes für das Fallgewicht, da Ausreisser nach oben häufiger sind

Luzerner Kantonsspital (LUKS), Austritte 2015 (Codiert bis 8.1.2015) als solche nach unten, welche zudem durch den Kurzliegerabschlag aufgefangen werden. Für jede DRG wird eine Normliegedauer berechnet und sogenannte «Outliers» definiert, bei deren Über-, resp. Unterschreiten der Fallpreis angepasst wird. Basierend auf den Daten der jeweils letzten zwei Jahre werden diese Werte jährlich neu berechnet und der Algorithmus des Groupers (entsprechende Gruppierungssoftware) adaptiert, so dass die gleiche Behandlung im neuen Grouper einer anderen DRG zugeordnet wird. 2015 werden in unserer breit diversifizierten Stichprobe ${ }^{1} 49 \%$ der Fälle einer anderen Gruppe zugeordnet.

\section{Hospitalisationsdauer hält mit dem Wirtschaftswachstum Schritt}

Ab 2016 kommt die Grouperversion 5 zum Einsatz. Deren Auswirkungen wurden analysiert. Dabei haben die implizit wirkenden Anpassungen der vorgegebenen Hospitalisationsdauer eine grössere Wirkung als die tendenziell nach unten korrigierten Kostengewichte. In unserer Stichprobe von 39260 Fällen aus dem Jahr 2015 aus dem Luzerner Kantonsspital mit einer Berücksichtigung von über 820 DRG liegt das Verhältnis von impliziter zu expliziter Senkung der Vergütung bei 4:1. Unmittelbar sichtbar wird die Vergütung durch Senkung des Fallgewichtes (cost weights) pro DRG reduziert. Die nicht unmittelbar sichtbare Senkung der Vergütung wird durch die Reduktion der zugestandenen Hospitalisationstage erreicht, welche konsekutiv den Leistungsdruck erhöht, d.h. die Spitäler haben nun für eine Behandlung nicht mehr durchschnittlich 7,4 Tage (2014), sondern 3\% weniger Zeit zur Verfügung. Angesichts der Tatsache, dass mit denselben Ressourcen an Personal, Material und Immobilien mehr Pa- 


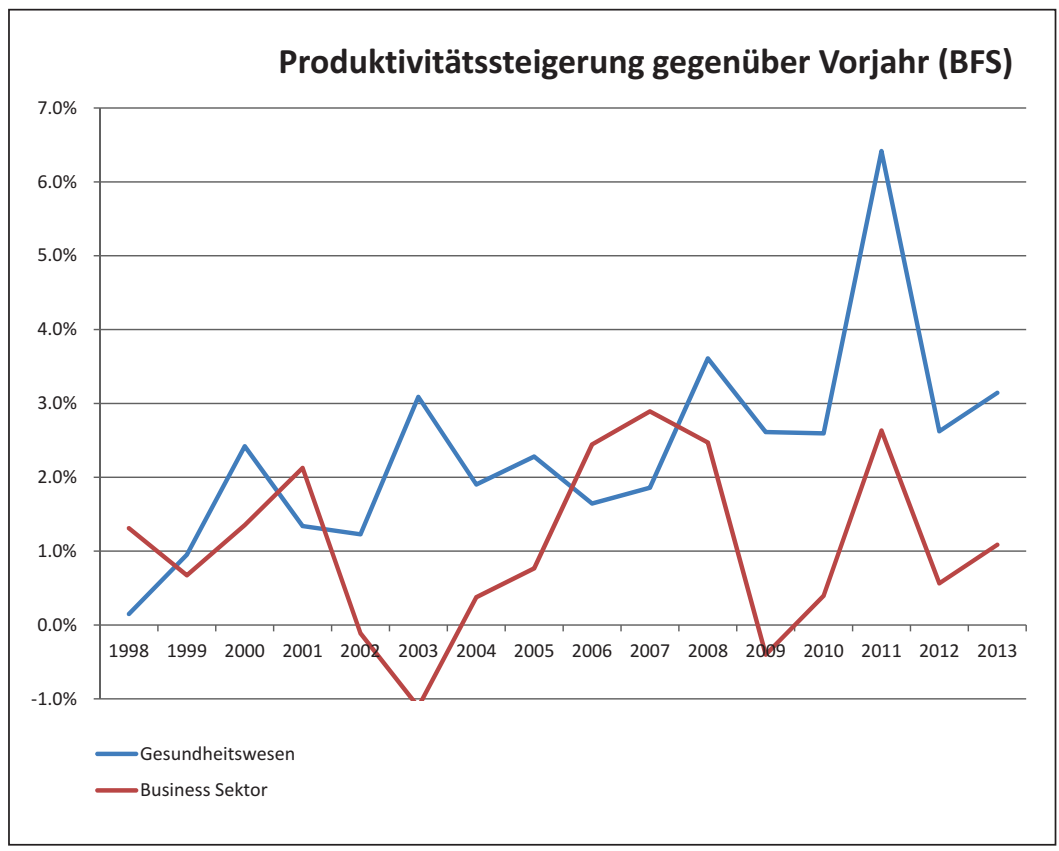

Abbildung 1: Jährliche Produktivitätssteigerung des Gesundheitswesens (ohne Heime und Sozialwesen) im Vergleich zu den anderen Branchen der Schweizer Volkswirtschaft. Dargestellt ist der Durchschnitt über 50 Branchen (orange) gegenüber dem Gesundheitssektor (blau).

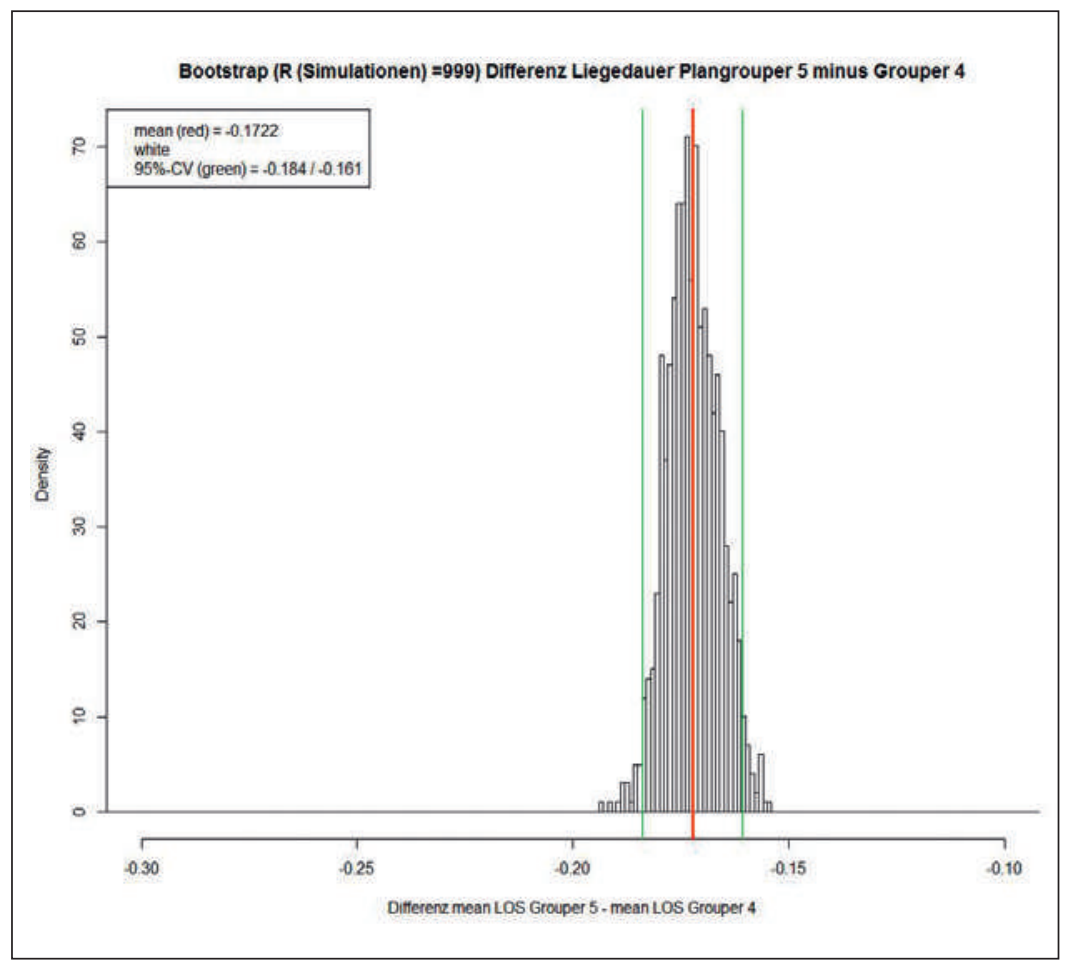

Abbildung 2: Die Bootstrap-Analyse, bei welcher mit - in unserem Fall 999 - Simulationen die statistisch möglichen Ergebnisszenarien berechnet werden, demonstriert die Auswirkungen des neuen SwissDRG-Groupers auf die Normliegedauer in Tagen (generiert aus der Stichprobe des Luzerner Kantonsspitals N = 39260). Das 95\%-Vertrauensintervall liegt zwischen $3 \%$ und 2,6\%, d.h. in 95\% der Szenarien wird die Reduktion zwischen $3 \%$ und $2,6 \%$ liegen. tienten behandelt werden müssen, ist dies zweifelsfrei sehr ambitiös. In anderen Worten widerspiegelt die zur Verfügung stehende Zeit einer Behandlung die Produktivität, so dass die Hospitalisationsdauer stellvertretend als Messgrösse für die Produktivität der Spitäler verwendet werden darf. In den Jahren 2009 bis 2014 wuchs die Schweizer Wirtschaft mit 0,2\% [1] jährlich. Von 2011 bis 2014 sank die Hospitalisationsdauer jährlich bereits um 0,99\%. Sie hielt also mit dem Wirtschaftswachstum Schritt.

2014 wurden in der ganzen Schweiz 1168684 [2] stationäre Patienten aus akutsomatischer Behandlung, welche nach SwissDRG abgerechnet wurde, entlassen. Die Kürzung der Normhospitalisationsdauer führt damit zu einer impliziten Erlösminderung der Spitäler bei gleichbleibender Leistung von ca. 325 Mio. CHF bei einer konservativ geschätzten Baserate von $9250 \mathrm{CHF}$, sofern die Spitäler die neue verkürzte Normhospitalisationsdauer und eine kompensatorische Zunahme der Behandlungsfälle nicht realisieren können.

\section{Produktivitätssteigerung im Gesund- heitssektor über Branchendurchschnitt}

Der Gesundheitssektor hat gemäss dem Bundesamt für Statistik (BFS) [3] in den letzten 16 Jahren in den meisten Jahren eine Produktivitätssteigerung realisiert, die über dem Branchendurchschnitt lag (Abb. 1). Gleichzeitig stiegen die Anforderungen an die Qualitätsadministration und Rechnungsstellung, für welche spezialisierte Mitarbeiter eingestellt werden mussten. Die regulatorischen Anforderungen nehmen auch in weiteren Bereichen wie beispielsweise dem klinischen Strahlenschutz zu. Damit entstehen den Spitälern zusätzliche nicht produktiv wirkende Kosten (siehe Abb. 1).

Die durch die zentrale Steuerung erzwungene Produktivitätssteigerung entbehrt der transparenten Herleitung. Für die Datengrundlage werden nicht alle Datensätze der (Daten liefernden) Netzwerkspitäler berücksichtigt, und «nicht plausible» Fälle werden eliminiert. Dieses Vorgehen als korrekt zu bezeichnen ist sowohl aus statistischer als auch betriebswirtschaftlicher Perspektive eher gewagt.

\section{Alle Spitäler stehen gleichsam unter erheblichem Produktivitätsdruck}

Die Vermutung liegt nahe, dass die Grouperversionen nicht nur basierend auf seriösen betriebswirtschaftlichen Basisdaten gesteuert, sondern auch durch politische Interessen beeinflusst werden. Die kaum unmittelbar sichtbare Kostensenkung eignet sich dazu 
hervorragend, da sie wenig transparent und nur mit einem gewissen Aufwand zu bestimmen ist. Die Senkungen der Kataloghospitalisationstage (Normliegedauer) stellen für die Spitäler einen mittelbaren, nicht direkt ersichtlichen Produktivitätszwang, resp. eine verdeckte Ertragseinbusse dar. Die sehr kleine Streuung im Diagramm der Abbildung 2 bedeutet, dass alle DRG in ähnlicher Ausprägung betroffen sind. Dies bedeutet auch, dass alle Spitäler gleichartig einem erheblichen Produktivitätsdruck ausgesetzt werden. Das System sieht für Patienten, welche berechtigterweise über die Normliegedauer hospitalisiert werden müssen, einen Tageszuschlag vor. Demgegenüber wird für Patienten, welche das Spital entsprechend früher verlassen können, pro Tag ein Abzug gemacht. Der neue Grouper gewährt den Zuschlag für Langlieger im Durchschnitt 6 Tage später als der vorjährige Grouper. Es ist unabdingbar, dass in Zukunft die Auswirkungen der jährlichen Systemanpassungen transparenter ausgewiesen werden. Vielfach wurde mit der DRG-Einführung vor Effekten auf das Personal gewarnt. Auf grund der vorliegenden Analyse werden zwei relevante Mechanismen aufgezeigt, die eine erhebliche Produktivitätssteigerung und eine relevante Erlösminderung bewirken. Wie sich dies auf die Mitarbeiter auswirkt, gilt es in den kommenden Jahren zu beachten.

\section{Forderung nach höheren Basisfallpreisen nicht systemkonform}

Wenn viele Standardbehandlungen unter erheblichen Produktivitätsdruck über Senkung der Normverweildauer und des Kostengewichtes geraten, so führt dies $\mathrm{zu}$ Aufwertungen anderer DRGs. Es ist daher nicht systemkonform, wenn gewisse Spitäler aufgrund ihrer spezifischen Versorgungscharakteristik für sich zusätzlich höhere Basisfallpreise fordern, weil unterschiedliche Patientenkategorien ja durch die Fallgewichtung repräsentiert werden sollten.

Bildnachweise

Grafiken zVg vom Autor

\section{Referenzen}

1 Gerhard Schwarz: Der Wohlstand in der Schweiz wächst doch. NZZ, 31. Dezember 2015, Seite 29.

2 Zahlenmaterial: Bundesamt für Statistik, Spitalbehandlung nach Alter und Geschlecht, http://www.bfs.admin.ch/bfs/portal/de/ index/themen/14/04/01/key/inanspruchnahme.html

3 Zahlenmaterial: Arbeitsproduktivität nach Branchen: http://www. bfs.admin.ch/bfs/portal/de/index/themen/04/03/blank/key/02. htm 